\title{
O PSICÓlOGO NAS POLÍTICAS PÚBLICAS A PARTIR DA CORRELAÇÃO ENTRE A CLÍNICA AMPLIADA E A REDUÇÃO DE DANOS
}

Rafael Bianchi Silva ${ }^{i} \oplus 000-0002-1170-7920$ Universidade Estadual de Londrina - UEL

Luiza Farias Miani ${ }^{\text {ii }}$ 0000-0003-1948-5661 Universidade Estadual de Londrina - UEL

RESUMO: O presente artigo objetiva percorrer a concepção de clínica ampliada e redução de danos com o intuito de acrescentar formas de pensar e atuar que divergem das práticas tradicionais no contexto de trabalho do psicólogo. A partir disso, construiremos aproximações dessas propostas quanto ao cuidado integral em saúde, pensando nestas estratégias enquanto formas de permitir a garantia dos direitos dos usuários e sua autonomia/ protagonismo. Tomaremos enquanto estratégia de pesquisa um estudo de caráter qualitativo, e como instrumento de coleta de dados utilizamos a Análise Documental, com o objetivo de descrever e representar o conteúdo dos documentos de uma forma que garanta a retomada de informações e a possibilidade de correlações entre os conteúdos. Conclui-se que, a concepção de clinica ampliada e as práticas de redução de danos se aproximam em alguns aspectos, principalmente nas suas concepções de sujeito/usuário. Quanto ao trabalho do psicólogo, entendemos que ambas as perspectivas podem potencializar problematizações e ampliações dos modos de atuação de forma a produzir ações mais participativas e inclusivas.

PALAVRAS-CHAVE: Clínica Ampliada. Redução de Danos. Psicologia

\section{THE PSYCHOLOGIST IN PUBLIC POLICIES FROM THE CORRELATION BETWEEN THE EXPANDED CLINIC AND DAMAGE REDUCTION}

ABSTRACT: This article aims to go through the concept of expanded clinic and harm reduction in order to add ways of thinking and acting that differ from traditional practices in the psychologist's work context. From this, we will build approximations of these proposals regarding comprehensive health care, thinking about these strategies as ways to allow the guarantee of users' rights and their autonomy / protagonism. We will take as a research strategy a qualitative study, and as a data collection instrument we use Document Analysis, with the aim of describing and representing the content of the documents in a way that guarantees the recovery of information and the possibility of correlations between the contents. It is concluded that, the concept of expanded clinic and harm reduction practices are similar in some aspects, mainly in their conceptions of subject / user As for the work of the psychologist, we understand that both perspectives can potentiate problematizations and extensions of the ways of performance in order to produce more participatory and inclusive actions.

KEYWORDS: Expanded Clinic. Harm Reduction. Psychology. 


\section{Introdução}

Historicamente, a Psicologia brasileira esteve atrelada aos interesses de uma classe elitista, voltada à prática de uma clínica individual. $\mathrm{O}$ desenvolvimento e disseminação das "psicologias" estiveram conectados ao surgimento do capitalismo como modelo político e econômico na sociedade. Utilizada como produtora de conhecimentos, a ciência psicológica contribuiu para a regulação e , controle e vigilância da população, estruturando procedimentos de disciplinamento de comportamentos, produzindo o modo "indivíduo" de subjetivação e modelos de intervenções psicológicas colocadas em prática nas políticas públicas nascentes no Brasil para a tentativa de moldar o cotidiano dos sujeitos (SILVA; CARVALHAES, 2016).

A partir desse contexto, torna-se importante a produção de práticas psicológicas que se apresentem como forças de resistência no sentido de produzir e dar visibilidade às múltiplas maneiras como as pessoas experimentam a vida, valorizando, com isso, a construção de modos singulares de viver.

De acordo com Carvalho e Yamamoto (2002), quando consideramos a atuação da Psicologia no âmbito da saúde, mesmo que anteriormente o psicólogo estivesse envolvido com os hospitais psiquiátricos e serviços ambulatoriais de saúde mental, foi apenas no final da década de 1970 que esses profissionais se inseriram nos serviços públicos de saúde de modo mais efetivo, com o objetivo de desenvolver modelos alternativos ao hospital psiquiátrico.

Além disso, os autores apresentam duas justificativas para o envolvimento dos psicólogos com as políticas públicas. A primeira se refere à crise econômica pela qual o Brasil estava passando, afetando, desse modo, o mercado dos atendimentos privados e, segundo, como resposta às críticas que a Psicologia clínica recebia por significar um não envolvimento da Psicologia com as questões sociais. No que se refere à reorganização da prática do psicólogo, foi somente nos anos 2000 que foi possível observar impactos significativos:

[...] As ações estão relacionadas ao evoluir da reforma psiquiátrica que, mais que propor reformas na saúde mental, criou um corpo teórico/prático que tem subsidiado o trabalho. Emergem temas como clínica ampliada, acompanhamento terapêutico, apoio matricial, filosofia da diferença, humanização, entre outros, que têm constituído um "modelo" de atuação (YAMAMOTO; OLIVEIRA, 2010, p. 16).

Conforme Curvo et al. (2018), o movimento da Reforma Psiquiátrica, buscou superar modelos de atenção à saúde fundamentados no modelo biomédico que, baseado em uma concepção positivista de saúde, estabelece uma relação hierárquica entre profissional, cuidador, usuário do serviço e mantém o profissional como detentor do saber e o usuário do serviço 
enquanto passivo de cuidados. Em oposição a isso, o movimento da Reforma Psiquiátrica propõe formas alternativas de cuidado, uma visão de saúde mental ampla, a desinstitucionalização da loucura, e a retirada do enfoque da doença do sujeito para o sujeito em sua experiência de sofrimento, em toda sua complexidade e integralidade.

A partir disso, ocorre uma alteração do foco que passa da cura de doenças para a prevenção e promoção da saúde enquanto um direito. Com isso, consolida-se ações fundamentadas nas demandas dos sujeitos de modo a promover a garantia de direitos e sua autonomia (LOPES; GONÇALVES, 2018). Os psicólogos passam, então, a se inserir em debates voltados para a compreensão das necessidades dos usuários e famílias no contexto das políticas públicas e a trabalhar com populações pobres.

[...] a Psicologia manteve-se cega e, assim, esteve incapaz, por muito tempo, de abordar a experiência de desenvolvimento de pessoas nessas condições e contextos, respondendo a essa condição sem uma adequada epistemologia e metodologia para sua compreensão e propostas de ação. [...] Daí decorre a importância da Psicologia no estudo e aprofundamento das questões sociais e psicossociais para o enfrentamento das desigualdades sociais. (GUZZO, 2016, p. 4).

Enquanto conceito, a noção de política pública refere-se a medidas e ações do governo que são geralmente dirigidas aos problemas de uma determinada população na tentativa de conduzir soluções, relacionada ao Estado. Tratam-se de "ações do Estado utilizadas para mediar conflitos e mazelas da sociedade, visando alcançar, como supracitado, o bem-estar da sociedade" (MOTA; SILVEIRA, 2018, p. 2).

Com a inserção nesse campo, emerge um debate sobre o Compromisso Social da Psicologia, concepção que se estabelece a partir de uma relação de forças situadas em um campo historicamente determinado, isto é, a partir das transformações da sociedade brasileira que impulsionaram a Psicologia a romper com seu modo de atuação:

[...] o debate referente às políticas públicas - que se evidencia como o mais antigo no que diz respeito ao Compromisso Social da Psicologia - mostra-se ainda recente para a profissão, e bastante complexo. Seguem sendo necessárias as problematizações não apenas sobre como entrar ou permanecer no campo, mas sobre o que fazer e como fazer estando nele (MIRON; GUARESCHI, 2017, p. 359).

Além disso, as autoras afirmam que o discurso de um Compromisso Social da Psicologia apresenta dois lados: um engajamento político da Psicologia com as transformações sociais, consolidando um sentido politicamente correto com relação às antigas práticas e, um interesse 
DOI 10.33872/revcontrad.v2nl.e018 | v. 2, n. 1 | Jan./Jun. 2021

em ampliar o campo de atuação profissional. Torna-se necessário então, problematizar essa ambivalência para acessar o compromisso ético e político da Psicologia com as transformações sociais.

A incorporação do psicólogo às questões sociais nos leva a tomar como ponto de partida nas reflexões que segue formas de atuação a partir de uma perspectiva biopsicossocial, pensando em um cuidado integral em saúde. Uma dessas expressões encontra-se no movimento pela clínica ampliada; outra, na estratégia de redução de danos, que se destaca enquanto prática de cuidado frente aos problemas decorrentes do uso de drogas.

Alguns estudos trazem o impacto da atuação do psicólogo em sua participação em uma clínica ampliada. Sundfeld (2010), por exemplo, discute como a inserção da Psicologia nos Núcleos de Apoio à Saúde da Família (NASF) pôde contribuir para a efetivação de uma clínica ampliada na rede básica, criticando modelos ditos saudáveis - que, na maioria das vezes, configuram-se como ortopédicos e modeladores -, permitindo a abertura dos usuários a experimentações. Além disso, aponta que esse modo de atuação trouxe provocações ao cotidiano, a fim de que equipe de trabalho dialogasse, questionasse e se fortalecesse, para afirmar formas de existir saudáveis e livres de padrões preestabelecidos.

Por sua vez, Eichenberg e Bernadi (2016) apontam que a prática do psicólogo na atenção básica em saúde mental por meio da estratégia da clínica ampliada caminha no sentido de romper com os velhos paradigmas da psicologia tradicional, estando mais abertos e envolvidos no trabalho interdisciplinar, na prevenção e promoção da saúde mental, na abordagem psicossocial, no trabalho em rede, e na singularidade e coletividade de outra forma de fazer clínica. Portanto, a prática do psicólogo na atenção básica em saúde mental pode acontecer sem a presença de protocolos prontos ou espaço físico especifico, além disso, a escuta do psicólogo pode acontecer no território onde as relações se constituem.

Em especial, com a disseminação da epidemia de HIV, a política de Redução de Danos foi incorporada em diversos países, incluindo o Brasil, visando o controle de sua transmissão entre usuários de drogas injetáveis (UDI). Enquanto premissa, as estratégias de RD objetivam a minimização de riscos e danos associados ao uso de drogas.

A legislação brasileira garante que os usuários de drogas tenham acesso universal aos serviços de saúde, isso inclui um sistema de assistência que deve ser oferecido em todos os níveis de atenção. A partir disso, a política se instaura em dispositivos extra-hospitalares, como no Centros de Atenção Psicossocial para Álcool e Drogas (CAPS ad) e serviços de atenção primária. (INGLEZ-DIAS et al., 2014) 
Os fundamentos dessa prática incluem uma estratégia de cuidado que vise a prevenção e garantia de informação para os usuários, bem como, uma compreensão ampla a respeito do fenômeno do consumo de drogas na sociedade, pautada na pluralidade e complexidade dos sujeitos. Parte-se ainda do princípio de que o indivíduo tem o direito de ser respeitado a partir de seus desejos e escolhas, garantindo assim, sua autonomia. (CÉZAR; OLIVEIRA, 2017). Posto isso, podemos observar que tanto a clínica ampliada quanto a redução de danos, adotam práticas que visam produzir um cuidado integral para os usuários, com serviços de saúde que garantam seus direitos e sua autonomia.

Tendo por pano de fundo os elementos discutidos até o momento, o presente artigo tem o objetivo de percorrer as concepções de clínica ampliada e redução de danos com o intuito de produzir formas de pensar a atuação do psicólogo no âmbito das políticas públicas. A partir disso, construiremos aproximações dessas propostas quanto ao cuidado integral em saúde, pensando nestas estratégias enquanto formas de permitir a garantia dos direitos dos usuários e sua autonomia/ protagonismo.

\section{Metodologia}

Enquanto estratégia de pesquisa, foi realizado um estudo teórico de caráter qualitativo compreendido enquanto "[...] um conjunto de diferentes técnicas interpretativas que visam descrever e decodificar os componentes de um sistema complexo de significados. Ela tem por objetivo traduzir e expressar os sentidos dos fenômenos do mundo social.” (NEVES, 1996, p.1).

Foi realizada uma análise documental que se constitui uma técnica importante na pesquisa qualitativa, seja complementando informações obtidas por outras técnicas, seja desvelando aspectos novos de um tema ou problema (LUDKE; ANDRÉ, 1986). A análise dos documentos tem por objetivo descrever e representar o conteúdo dos documentos de uma forma distinta da original, visando garantir a recuperação da informação nele contida e possibilitar seu intercâmbio, difusão e uso (SOUZA; KANTORSKI; LUIZ, 2011, p. 222).

Utilizou-se como base de dados, artigos e publicações da área a partir dos quais foi realizado um estudo histórico da atuação da Psicologia e seu envolvimento com as questões sociais, especialmente no âmbito das políticas públicas, percorremos ainda, o conceito de Compromisso Social da Psicologia. Em um segundo momento, analisou-se as práticas psicológicas relacionadas às estratégias da clínica ampliada e redução de danos, em relação a uma visão de integralidade no cuidado em saúde dos usuários e que possam contribuir para o protagonismo e autonomia do usuário por meio de um cuidado integral em saúde. 


\section{Cuidado integral: como a clínica ampliada e a política de redução de danos contribuem para o protagonismo dos usuários}

O direito universal à atenção integral à saúde é uma conquista que remete ao período de redemocratização do país após cerca de duas décadas sob regime de Ditadura Civil Militar (1964-1985). Segundo Curvo et al. (2018), vale ressaltar que, o modelo de atenção à saúde brasileira estava vinculado ao sistema previdenciário, privilegiando os trabalhadores que contribuíram com os fundos de previdência, deixando excluída parte da população rural e urbana, como agricultores, autônomos, trabalhadores informais, entre outros. Na segunda metade dos anos 1970 surgem narrativas que questionavam tal modelo de assistência à saúde, dentre eles, a Reforma Sanitária e posterior Reforma Psiquiátrica, as quais aglutinaram movimentos populares com movimentos de acadêmicos, técnicos dos serviços e partidos políticos de esquerda e centroesquerda, em torno da luta pela saúde como um direito de todos.

Para Baptista (2005), após anos de discussões sobre o tema, a VIII Conferência Nacional de Saúde (CNS) - ocorrida em 1986 - e a criação do Sistema Único de Saúde (SUS) em 1988, ampliaram o conceito de saúde que deixa de ser vista na perspectiva saúde-doença (e, portanto, necessitando de uma cura) e passa a ser vista em suas determinações sociais, extrapolando o clássico modelo biologicista. Para além disso, o SUS passou a implementar a descentralização e hierarquização dos serviços, a universalidade de acesso à saúde e a importância da integralidade no cuidado.

Assim como o conceito de saúde incorporou os aspectos biopsicossociais do contexto de vida dos sujeitos, o modelo de atenção à saúde do SUS também passou a considerar o sujeito como um ser integral que demanda ações de cuidado articuladas multidisciplinarmente, intra e intersetorialmente, ou seja, que garantam a integralidade das ações nas três esferas de atenção à saúde (primária, secundária e terciária), e articuladas a outras políticas públicas.

Curvo et al. (2018) afirma que podemos perceber a integralidade como dimensão das práticas profissionais que se faz pelo encontro/relação entre profissionais e usuários na busca por formas mais abrangentes possíveis de atender às necessidades deste usuário, de modo a gerar e aproveitar as oportunidades para fortalecimento de vínculo e produção do seu protagonismo. Desse modo, os processos de trabalho devem incluir os sujeitos e seus coletivos nas decisões terapêuticas, ações preventivas e de promoção de saúde de determinado território. Porém, ao compreender a complexidade das demandas se faz necessário articular os diversos saberes em prol de respostas mais eficazes para suas demandas. Entra em cena o trabalho transdisciplinar e multiprofissional das equipes de saúde na construção do cuidado integral, 
bem como, torna-se necessária a articulação com a rede de saúde, dispondo dos diversos serviços que sejam importantes para o caso.

Nessa direção, entendemos o cuidado integral para além de um conjunto de práticas e procedimentos necessários que compõem um determinado tratamento. Consoante com esse ponto, Mattos (2013) afirma que o cuidado integral deve ser permitido ao se pensar constantemente em novas formas de agir em saúde, ou seja, pensar a própria forma de organização dos processos de trabalho dos serviços públicos de saúde. Trata-se, então, da organização contínua do processo de trabalho de modo que se ampliem as possibilidades de apreensão das necessidades dos sujeitos que buscam os serviços, tendo o diálogo entre o sujeito cuidado e o sujeito cuidador como central para que tal apreensão aconteça. Esse diálogo tornase então o ponto de partida e deve ocorrer entre os diferentes sujeitos envolvidos e as diferentes formas de perceber as necessidades dos serviços de saúde.

Segundo Campo et al. (2014), a clínica ampliada e compartilhada é um conceito desenvolvido por Gastão Wagner sendo incorporado aos discursos oficiais do Ministério da Saúde a partir dos anos 2000, especialmente pelas políticas de Atenção Básica e de Humanização. Integrada a um conjunto mais amplo de propostas, caracterizado como metodologia Paideia, a Clínica Ampliada é um dos três eixos de aplicação dessa metodologia, junto com o Apoio Matricial e o Apoio Institucional. Esse método de gestão teve como objetivo a criação nos locais de atenção à saúde, espaços de cogestão, contribuindo para a formação de sujeitos críticos e implicados no desenvolvimento das instituições, atuando sobre relações de poder, produção de conhecimento sobre si, sobre os outros e sobre os contextos de vida.

Ainda, segundo Campos et al. (2014) desenvolvimento de uma gestão compartilhada do cuidado, visa amenizar a desigualdade de saber-poder na relação profissional de saúde-usuário, produzindo práticas de cuidado que se pautem mais no diálogo e na negociação do que em saberes prescritivos. Busca-se, dessa forma, a escuta, o acolhimento dos afetos envolvidos na relação entre profissionais e usuários, primando por uma relação mais horizontalizada, em que o usuário assume um papel mais ativo de seu cuidado.

Coerente com o princípio da integralidade a estratégia da clínica ampliada opera uma tripla ampliação do cuidado: do objeto, do objetivo e dos meios de trabalho. Quanto ao primeiro ponto, nessa perspectiva, o objeto de trabalho de qualquer profissional de saúde deve ser a pessoa ou grupos de pessoas, convidando a um desafio de trabalho para que pessoas se responsabilizem por pessoas.

A clínica ampliada pretende a ampliação do objeto de trabalho dos profissionais, ao incorporar as fragilidades subjetivas e as redes socais para além dos riscos biológicos 
(CAMPOS et al., 2014). Isso implica no trabalho com pessoas e coletivos considerando as suas variáveis e características singulares (como, por exemplo, a capacidade para elaboração de interpretações e posicionamentos, de estabelecer contratos e compromissos).

Importante destacar que não se desconsidera a doença ou seus riscos, mas assumindo que estes, embora mantenham padrões e regularidades, são influenciados por dimensões como os aspectos cultural e socioeconômico. Desta maneira, tanto a prevenção como a terapêutica, proposta pela clínica ampliada, partem das evidências e da avaliação de riscos para negociar com as pessoas o que é possível no caso particular, para este paciente, no contexto vivido e no tempo histórico presente.

No que se refere ao segundo ponto, Campos et al. (2014) coloca que a clínica ampliada contempla a necessidade da extensão dos objetivos do trabalho dos profissionais, os quais, além de buscar a cura, reabilitação e prevenção de danos em pessoas doentes, devem produzir saúde, o que significa apoiar o desenvolvimento de maiores graus de autonomia e autocuidado dos sujeitos, de sua capacidade de agir, pensar e desenvolver novas formas de vida para si e modos de posicionamento diante da sua saúde e do seu contexto. Dessa forma, o objetivo da clínica ampliada é colocar primeiro plano a situação real do trabalho em saúde, vivida a cada instante por sujeitos reais abarcados por suas complexidades.

Além disso, a respeito do terceiro ponto, os meios de trabalho também devem ser modificados, sendo necessários arranjos que privilegiem uma comunicação transversal na equipe e entre equipes, bem como técnicas relacionais que permitam uma clínica compartilhada, dentre elas: "a capacidade de escuta do outro e de si mesmo, a capacidade de lidar com condutas automatizadas de forma crítica, de lidar com a expressão de problemas sociais e subjetivos, com família e com comunidade etc" (BRASIL, 2009, p. 17). Segundo Sampaio e Freitas (2012), os profissionais devem estimular a participação dos sujeitos envolvidos, aproximando-os de uma ação crítica e política na qual seja possível protagonizar as diversas cenas no campo da saúde e da cidadania. Dessa forma, a clínica ampliada implica na construção de uma responsabilidade compartilhada com os sujeitos envolvidos, sendo que:

[...] o reconhecimento da complexidade deve significar o reconhecimento da necessidade de compartilhar diagnósticos de problemas e propostas de solução. Este compartilhamento vai tanto na direção da equipe de saúde, dos serviços de saúde e da ação intersetorial, como no sentido dos usuários (BRASIL, 2008, p.15).

A clínica ampliada pretende a autonomia e emancipação do sujeito, ou seja, privilegia uma ação profissional que busque a saída do usuário da condição de passividade para a 
DOI 10.33872/revcontrad.v2nl.e018 | v. 2, n. 1 | Jan./Jun. 2021

consciência da realidade e posse de uma postura ativa. Assim, ela possibilita que outros aspectos do sujeito, que não apenas o biológico possa ser compreendido e trabalhado pelos profissionais, permitindo a tomada de consciência dos seus direitos, possibilitando um trabalho de reflexão sobre as próprias condições e de luta pelos os próprios direitos, consequentemente a ampliação do grau de autonomia do usuário, da família e da comunidade.

Percorrido de forma breve histórico da clínica ampliada, apontando seus objetivos e sua relação com o cuidado integral em saúde, passaremos a seguir para uma contextualização da política de redução de danos de modo a apresentar seus princípios e fundamentos.

Inicialmente, a redução de danos (RD) era vista socialmente como uma prática de incentivo ao uso de substâncias psicoativas e, portanto, vista negativamente por muitos profissionais da área da saúde. No começo, práticas como a troca de seringas foram avaliadas como um meio para os usuários permanecerem inseridos na dependência química, proporcionando assim, um afastamento do objetivo central das políticas de saúde, voltadas para a abstinência (DOMANICO, 2019). Devido a isso, a RD, enquanto movimento, foi se desenvolvendo à margem do SUS e desarticulada de outras ações e proposições colocadas em prática pela política pública, encontrando forte tensionamento por parte de diferentes setores do aparelho estatal, posicionados a favor de uma política antidrogas (FONSÊCA, 2012).

De acordo com Marlatt (1999), existem cinco princípios básicos da redução de danos associados ao uso de drogas. Primeiramente, a RD é uma alternativa de saúde pública para modelos moral/criminal e de doença do uso e da dependência de drogas. Em segundo lugar, reconhece a abstinência como resultado ideal, mas aceita alternativas que reduzam danos. $\mathrm{O}$ terceiro princípio afirma que ela se trata de uma abordagem de "baixo para cima", ou seja, baseada na defesa do usuário, em detrimento da ação do Estado. Em quarto lugar, promove acesso a serviços de baixa exigência como alternativa para abordagens tradicionais de alta exigência. E, por fim, baseia-se nos princípios do pragmatismo empático versus idealismo moralista. A respeito desses princípios, Fonsêca (2012, p. 22-23) afirma que:

[...] percebe-se que o princípio fundamental que orienta a Redução de Danos é o respeito à vida e a liberdade de escolha, possibilitando que sua atuação se apoie na promoção do exercício e respeito às diferenças, retomada do acesso à dignidade e à cidadania, uma abordagem humana para os que estão em maior risco e atuação preventiva junto às populações ainda não atingidas são algumas das atribuições desse novo modelo que tenta se isentar de julgamentos crítico-morais, optando pela vida, pela saúde e responsabilidade pessoal $[\ldots]$ 
A luta antimanicomial e a militância a respeito da AIDS propiciaram importantes avanços para a instalação da política da RD no Brasil. Isso se deu, segundo Souza (2007), a partir da constituição de diferentes modelos de atenção à saúde, alteração de paradigmas morais vigentes e a consolidação de um plano de políticas para a saúde que fossem defensoras dos direitos das pessoas que usam drogas. Sobre esse contexto e o que foi potencializado a partir dele, Dias (2008, p. 76-77) acrescenta que:

[...] essa abertura operada pelo movimento sanitário foi de suma importância para a integralidade da rede de saúde e descentralização, regionalização, hierarquização dos serviços com a participação social na gestão dos mesmos. Tal direção da política pública permite que mais tarde as ações de RD estejam inseridas no SUS, como política de saúde para os usuários de drogas e em articulação com a saúde mental. Foi na abertura de espaços concretos de participação que as práticas de RD avançaram, com a criação de programas de saúde que são tributários, direta ou indiretamente, do movimento sanitarista e na defesa da saúde pública como "direito de todos".

O surgimento da $\mathrm{RD}$, a partir de seus princípios, fundamentos e práticas, acaba por ressignificar o próprio SUS, renovando uma visão de saúde articulada com a dimensão social. Partindo do conceito de saúde discutido pela Organização Mundial da Saúde (OMS) - o qual a caracteriza não somente a ausência de uma doença ou enfermidade, mas um estado completo de bem-estar físico, mental e social -, Almeida (2003, p. 56) afirma que:

Dentro do âmbito da saúde, a redução de danos ocupa um espaço de renovação do paradigma de atendimento, sendo uma ação que vem como alternativa à consolidação dos princípios da nova saúde pública, do Sistema Único de Saúde (SUS) e do conceito de saúde implicado nesse processo. [...] Esses conceitos nos levam a pensar que o primordial não é extinguir os sintomas presentes em uma pessoa, mas sim que esta consiga ter uma boa qualidade de vida, de acordo com suas possibilidades.

Notamos aqui, o fato de que a RD adota um conceito ampliado de saúde, deixando de significar apenas um estado de ausência de doença. Reconhece que a qualidade de vida pode ser ampliada a partir de estratégias que não necessariamente livram o usuário de uma doença, mas que sim, podem minimizar as consequências negativas advindas dessa condição.

Após apresentar esses dois universos, seguiremos com os comparativos de ambas as práticas, com o intuito de apresentar novas possibilidades de entendimento e atuação para os psicólogos que extrapola as práticas da clínica tradicional. O objetivo a seguir é identificar a existência de pontos em que as duas práticas possam se aproximar. 


\section{A RD e a Clínica Ampliada: o olhar para o usuário}

Souza (2007) afirma que o grande desafio da RD é o mesmo da democracia: garantir às minorias a possibilidade de expressão. Diante disso, surge o questionamento sobre quais instituições estariam a serviço de garantir que os usuários sejam os porta-vozes de sua própria política, ou seja, protagonistas em defesa da vida.

Atentamos aqui, para a importância dos conceitos de direito e autonomia levantados pela RD. As práticas da política defendem a perspectiva de que o sujeito escolha, dentro de suas alternativas, qual percurso quer seguir. Dessa forma, aparece a relevância de reconhecer o usuário como um cidadão de direitos o que implica ter acesso à informação, escolher seu objetivo e ser amparado por um serviço de saúde. Além disso, levanta-se a necessidade de reconhecer as pluralidades e compreender que o sujeito apresenta demandas particulares e tem o direito de ser acolhido a partir delas.

Pode-se estabelecer um enlace com a clínica ampliada, visto que a mesma reconhece o usuário como uma pessoa de direitos, o qual deve ser envolvido por medidas de saúde que visem a proteção dos mesmos. Ainda, a clínica ampliada lança um olhar para as diferenças e possibilita as diversidades, constituindo diversas conexões com as diferentes redes sociais (como movimento de mulheres, movimento gay, movimento de saúde mental, entre outros), objetivando aperfeiçoar a participação dos sujeitos envolvidos, aproximando-os de uma ação crítica e política na qual seja possível protagonizar as diversas cenas no campo da saúde e da cidadania (SAMPAIO; FREITAS, 2012).

Podemos observar que há, por meio do olhar ampliado, a necessidade de preparar os sujeitos para fazer valer os próprios direitos. Portanto, é importante não só a garantia de proteção, mas um trabalho árduo do sistema de saúde que possibilite o acesso dos usuários a participação social e autonomia. Um exemplo disso no contexto brasileiro são os Conselhos e as Conferências realizadas em diferentes políticas públicas que possibilitam participação democrática por meio da reunião de diversos segmentos representativos da sociedade em um espaço de debate para avaliar, propor diretrizes a respeito dos serviços ofertados (BRASIL, 2013).

Observa-se que as práticas da RD e da clínica ampliada enfatizam que a autonomia é uma importante propiciadora de condições que garantem o usuário enquanto protagonista de sua própria vida e, de certa forma, garantem também, a independência do sujeito frente aos problemas enfrentados. Assim, a autonomia funda-se como um conceito essencial para a atuação dos profissionais. Para além de um direito, é considerada uma potencialidade a ser 
atingida e desenvolvida no usuário que demanda ações de intervenção direcionadas para essa finalidade.

A atuação da redução de danos envolve o contato direto com a comunidade, desse modo, os redutores de danos valorizam o saber dos usuários, e a transmissão de informações para a comunidade através de discussões coletivas de modo a não restringir o planejamento de ações de intervenções aos profissionais da saúde, possibilitando, assim, que o usuário participe das decisões e se consolide uma autonomia e emancipação do usuário (GOMES; DALLA; VECCHIA, 2018).

Nessa perspectiva, os pressupostos da RD consideram necessário levar em conta a realidade de cada usuário, reconhecendo-o como um cidadão que possui o direito de receber informações e orientações e, a partir daí, decidir por si o que seria melhor para ele. Conforme os fundamentos da $\mathrm{RD}$, é necessário estabelecer vínculo com o usuário, facilitar o acesso a informações e orientações e estimular a ida ao serviço de saúde através da utilização de propostas diversificadas e construídas com cada usuário e sua rede social. Considera-se:

[...] o que é pedido e as possibilidades para compor um acompanhamento, com combinações em comum acordo. Fundamentando-se na autonomia do usuário, na sua liberdade de escolha e nos princípios de cidadania e direitos humanos. A flexibilidade permite um diálogo maior com diferentes instâncias, ressituando o debate das drogas pautado pela ética e pelo respeito às subjetividades (FONSÊCA, 2012, p. 13-14).

Conforme Campos et al. (2014), esse estabelecimento do diálogo na relação profissional-usuário também está implicado na clínica ampliada, a qual identifica que o trabalho precisa ser realizado em uma lógica dialogal, na qual não há uma única verdade, mas o compartilhamento entre os saberes que irão auxiliar a construção de manejos do tratamento em relação a saúde do usuário. Nessa lógica, o espaço comunitário e os alicerces populares são valorizados, de forma a respeitar e interagir com os ditos e vivências que sustentam a constituição desses sujeitos.

Do mesmo modo, a redução de danos, ao permitir a flexibilidade no contrato com o usuário, pode possibilitar por meio dessas ações políticas, a valorização da emancipação do sujeito, ou seja, um movimento profissional que visa a saída do usuário da condição de passividade para a consciência da realidade e posse de uma postura ativa.

Os usuários devem ser vistos como cidadãos que possuem múltiplos modos de interagir com o mundo o que implica, dessa forma, diferentes maneiras de construir sua própria dimensão subjetiva. Com isso, é importante analisar as demandas dos usuários nas dimensões históricas, 
sociais pessoais e coletivas, para que, com isso, possam realizar uma intervenção psicológica mais efetiva. Sobre essa perspectiva:

Compreender o papel ativo do indivíduo e a influência das relações sociais, valores e conhecimentos culturais sobre o desenvolvimento humano pode favorecer a construção de uma atuação profissional que seja transformadora das desigualdades sociais. "Ao levar em consideração essa dimensão do desenvolvimento dos sujeitos, contribui-se para a promoção de novos significados ao lugar do sujeito cidadão, autônomo e que deve ter vez e voz no processo de tomada de decisão e de resolução das dificuldades e problemas vivenciados" (CREPOP, 2008, p. 23). Portanto, valorizar a experiência subjetiva do indivíduo contribui para fazê-lo reconhecer sua identidade e seu poder pessoal.

Retomando a conexão com a clínica ampliada, durante o contato com o usuário, é necessária uma análise das demandas concretas dos sujeitos (SILVA; BONATTI, 2019). Desse modo, é importante a expansão para uma avaliação das questões sociais e subjetivas dos usuários, baseando-se na escuta e reconhecimento de seus saberes, desejos, interesses, e questionando essa população sobre os sentidos daquilo que estão vivendo. Portanto, é necessário:

[...] acolher toda queixa ou relato do usuário mesmo quando aparentemente não interessar diretamente para o diagnóstico e tratamento. Mais do que isto, é preciso ajudá-lo a reconstruir e respeitar os motivos que ocasionaram o seu adoecimento e as correlações que o usuário estabelece entre o que sente e a vida - as relações com seus convivas e desafetos. Ou seja, perguntar por que ele acredita que adoeceu e como ele se sente quando tem este ou aquele sintoma. Quanto mais a doença for compreendida e correlacionada com a vida, menos chance haverá de se tornar um problema. Assim o usuário poderá perceber que, senão nas causas, pelo menos nos desdobramentos o adoecimento não está isolado da sua vida [...] (BRASIL, 2008, p. 24)

Sendo assim, os profissionais devem seguir uma escuta das demandas dos usuários, evitando recomendações pastorais e culpabilizantes. É necessário trabalhar a partir de ofertas e não apenas restrições. Entendendo, ainda, as especificidades de cada usuário e valorizando a qualidade de vida dos sujeitos. (BRASIL, 2010).

A partir dos pontos aqui levantados, percebe-se um alinhamento entre a clínica ampliada e a proposta da redução de danos visto que ambas se fundamentam como práticas mais negociada do que prescritiva, de modo a contribuir para um cuidado integral em saúde dos sujeitos. 
DOI 10.33872/revcontrad.v2nl.e018 | v. 2, n. 1 | Jan./Jun. 2021

\section{Considerações finais}

A partir da década de 1970, devido à crise econômica e decréscimo no mercado dos atendimentos privados, o psicólogo começa a se inserir nas Políticas Públicas, se deparando com demandas diversas que levaram a ampliação de seus horizontes de atuação clínica. Desenvolvem-se novos discursos que apontam para a análise das práticas psicológicas até então realizadas, aos mecanismos de normatização/normalização por elas produzidas. Além disso, esse processo propiciou uma maior proximidade quanto ao cuidado do usuário/população atendida nos diferentes serviços e a necessidade de um envolvimento da Psicologia com as questões sociais.

Com isso, os debates profissionais passaram a girar em torno da compreensão das necessidades dos usuários e seus familiares, na execução de ações visando à garantia de seus direitos, assim como a prevenção, promoção e proteção em saúde. Dessa forma, busca-se uma Psicologia mais voltada para os problemas concretos da realidade e preocupada quanto as questões sociais.

Diante dessas reflexões, a clínica ampliada se mostra como um conceito que permite um movimento de expansão de suas atuações. Traz consigo a ideia de uma prática clínica que envolve a construção de um olhar para com e entre os sujeitos, a preocupação em ampliar o atendimento clínico em diferentes contextos e, para além, uma diferente concepção da relação saúde-doença. Além disso, materializa uma clínica voltada para existência concreta dos sujeitos, incluindo os direitos básicos para a sobrevivência da pessoa atendida e sua família, a garantia de seus direitos e um trabalho que movimente o protagonismo desses usuários, enquanto participantes das políticas públicas.

Paralelamente à consolidação das ideias da clínica ampliada, outras práticas e conceitos foram surgindo e proporcionando a produção e utilização de novas perspectivas de atuação no âmbito da saúde. Uma delas é a política de redução de danos a qual observou-se alinhada com os pressupostos adotados por essa prática e os fundamentos da clínica ampliada. A RD está intimamente ligada à garantia dos direitos do usuário, à defesa da autonomia do usuário e do cumprimento de suas escolhas e desejos. Essa perspectiva de atuação e de usuário possibilita debates para os profissionais que vão atuar nesse cenário, como por exemplo o psicólogo, uma vez que rompe com lógicas culpabilizastes e discriminatórias.

Posto isso, podemos concluir que a clínica ampliada e a RD se assemelham em diferentes aspectos. Os elementos por elas trazidos ao campo da saúde contribuem para potencializar problematizações e ampliações dos modos de atuação do psicólogo no contexto 
DOI 10.33872/revcontrad.v2nl.e018 | v. 2, n. 1| Jan./Jun. 2021

das políticas públicas de forma a fomentar novos fazeres que possam produzir condições que ultrapassem a objetivação do usuário e estejam alinhadas a ações relacionadas a saúde integral do usuário.

\section{REFERÊNCIAS:}

ALMEIDA, C. B. Conceito de redução de danos: uma apreciação crítica. Boletim da Saúde, v. 17, n. 1, 2003. Disponível em: http://www.boletimdasaude.rs.gov.br/ conteudo/1228/conceito-de-reducao-de-danos:-uma-apreciacao-critica. Acesso em: 7 jun. 2020.

BAPTISTA, T. V. de F. O direito à saúde no Brasil: sobre como chegamos no Sistema Único de Saúde e o que queremos dele. In: Textos de apoio em políticas de saúde. Rio de Janeiro (RJ): Fiocruz, 2005.

BRASIL. Ministério da Saúde. Secretaria de Atenção à Saúde. Núcleo Técnico da Política Nacional de Humanização. Acolhimento nas práticas de produção de saúde. Brasília. 2008. Disponível em: http://bvsms.saude.gov.b r/bvs/publicacoes/ humanizasus_gestores_trabalhadores_sus_4ed.pdf. Acesso em: 12 jun. 2020.

BRASIL. Ministério da Saúde Política Nacional de Humanização da Atenção e Gestão do SUS. Clínica ampliada e compartilhada/Ministério da Saúde, Secretaria de Atenção à Saúde, Política Nacional de Humanização da Atenção e Gestão do SUS. Brasília, 2009. Disponível em: http://bvsms.saude.gov.br/bvs/ publicacoes/ clinica_ampliada_compartilhada. pdf Acesso em: 12 nov. 2020.

BRASIL, Ministério da Saúde (MS) Secretaria de Atenção à Saúde (SAS) Política Nacional de Humanização (PNH). Política Nacional de Humanização. Brasília, 2010. Disponível em: http://www.redehumanizasus.net/sites/default/files/diretrizes_e_dispositivos_da_pnh1.pdf

Acesso em: 30 nov. 2020.

BRASIL. Ministério da Saúde. Conselhos de saúde: a responsabilidade do controle social democrático do SUS. Brasília. 2013. Disponível em: http://bvsms.saude.gov.br/ bvs/publicacoes/conselhos_saude_responsabilidade_controle_2edicao.pdf. Acesso em: 22 jun. 2020.

CAMPOS, G. W. D. S. et al. A aplicação da metodologia Paideia no apoio institucional, no apoio matricial e na clínica ampliada. Interface-Comunicação, Saúde, Educação, v. 18, p. 983-995, 2014.

CARVALHO, D. B.; YAMAMOTO, O. H. Psicologia e políticas públicas de saúde: anotações para uma análise da experiência brasileira. Psicologia para a América Latina, v. 1, p. $1-12$, 2002. Disponível em: http://pepsic.bvsalud.org/ scielo.php?script=sci_a rttext\&pid=S1870-350X2002000100002\&lng=pt\&tlng. Acesso em: 25 jul. 2020

CENTRO DE REFERÊNCIA TÉCNICA EM PSICOLOGIA E POLÍTICAS PÚBLICAS. (CREPOP) Referência técnica para atuação do(a) psicólogo(a) no CRAS/SUAS / Conselho Federal de Psicologia (CFP). Brasília, CFP, 2007. (re-impressão 2008). Disponível em: http://www.crpsp.org.br/portal/comunicacao/artes-graficas/arquivos/2008-crepop-crassuas.pdf. Acesso em: 01 ago. 2020.

CÉZAR, M. de A.; OLIVEIRA, M. A. Redução de danos: uma experiência na atenção básica. Mental, v. $11 . \quad$ n. 21, p. 486-500, 2017. Retirado de: 
DOI 10.33872/revcontrad.v2nl.e018 | |v. 2, n. 1| Jan./Jun. 2021

http://pepsic.bvsalud.org/scielo.php?script=sci_arttext\&pid=S167944272017000200012\&lng=pt\&tlng=pt. Acesso em: 27 de out. de 2020.

CURVO, D. R. et al. Integralidade e clínica ampliada na promoção do direito à saúde das pessoas em situação de rua. Cadernos Brasileiros de Saúde Mental/Brazilian Journal of Mental Health, v.10, n. 25, 58-82, 2018.

DIAS, R. M. Do asilo ao CAPSad: lutas na saúde, biopoder e redução de danos. $158 \mathrm{f}$. Dissertação (Mestrado) - Universidade Federal Fluminense, Departamento de Psicologia, Instituto de Ciências Humanas e Filosofia, Niterói, 2008.

DOMANICO, A. História, conceito e princípios de redução de danos. In: SURJUS, L.; FORMIGONI, M.L.; GOUVEIA, F. (Org.). Redução de danos: conceitos e práticas. São Paulo: UNIFESP, p. 5-15, 2019. Disponível em: http://www.tjmt.jus.br/intranet.arq/ cms/grupopaginas/105/988/Redu\%C3\%A7\%C3\%A3o_de_danosconceitos_e_pr\%C3\%A1tica s.pdf Acesso em: 30 ago. 2020.

EICHENBERG, J. F.; BERNADI, A. B. A prática do psicólogo na atenção básica em saúde mental: uma proposta da clínica ampliada. Artigo produzido para conclusão do curso de Pós Graduação de Saúde Mental e Atenção Psicossocial do Centro Universitário para o Desenvolvimento do Alto Vale do Itajaí- Vale do Itajaí, SC. 2016.

FONSECA, C. J. B. Conhecendo a redução de danos enquanto uma proposta ética. Psicologia \& Saberes, Maceió, v. 1, n. 1, p. 11-36, 2012.

GOMES, T. B.; DALLA VECCHIA, M. Estratégias de redução de danos no uso prejudicial de álcool e outras drogas: revisão de literatura. Ciência \& Saúde Coletiva, v. 23, n.7, p. 23272338, 2018.

GUZZO, R. A (Des)Igualdade Social e A Psicologia: Uma Perspectiva Para o Debate Sobre a Pobreza. In: Psicologia e Pobreza: contribuições para uma análise psicossocial. Cap. 11. UFC, 2016. Disponível em: https://www.researchgate.net/ publication/305469045_A_DESIGUALDADE_SOCIAL_E_A_PSICOLOGIA_UMA_PERS PECTIVA_PARA_O_DEBATE_SOBRE_A_POBREZA_1 Acesso em: 13 set. 2020.

INGLEZ-DIAS, A. et al. Políticas de redução de danos no Brasil: contribuições de um programa norte-americano. Ciência \& Saúde Coletiva, v. 19, n. 1, p. 147-158, 2014.

LOPES, H. P.; GONÇALVES, A. M. A política nacional de redução de danos: do paradigma da abstinência às ações de liberdade. Pesquisas e Práticas Psicossociais, v. 13, n. 1, 2018. São João del Rei. Disponível em: http://www.seer.ufsj.edu.br/ index.php/revista_ppp/ article/view/2858. Acesso em: 10 set. 2020.

LUDKE, M.; ANDRÉ, M. E. D. A pesquisa em educação: abordagens qualitativas. $2^{\mathrm{a}}$ ed. São Paulo: EPU. 1986.

MATTOS, R. A. Cuidado Prudente para uma vida decente. In: Cuidado: as fronteiras da integralidade. Rio de Janeiro: CEPESC/UERJ, IMS: ABRASCO, 2013.

MARLATT, G. A. Redução de Danos: Estratégias Práticas Para Lidar com Comportamentos de Alto Risco. Porto Alegre: Artes Médicas, 1999.

MIRON, A. X.; GUARESCHI, N. M. D. F. Compromisso social da psicologia e sistema único de assistência social: Possíveis articulações. Psicologia: Ciência e Profissão, v. 37, n. 2, p. 349$362,2017$. 
MOTA, P. S.; SILVEIRA, H. F. D. Psicologia e políticas públicas em saúde: uma revisão integrativa. 2018. Disponível em: http://repositorio.unilab.edu.br:8080/ jspui/handle/ 123456789/1373 Acesso em: 15 jun. 2020.

NEVES, J. L. Pesquisa Qualitativa: características, usos e possibilidades. Caderno de pesquisas em Administração, São Paulo, v. 1, n. 3, 1996.

SAMPAIO, C.; FREITAS, D. S. Redução de danos e SUS: enlaces, contribuições e interfaces. In: Textos de Apoio a IV Conferência Nacional de Saúde Mental. 2012. Disponível em: https://site.mppr.mp.br/arquivos/File/Projeto_Semear/Temas_Revelantes/

Reducao_de_Danos_e_SUS.pdf Acesso em: 10 setembro 2020.

SILVA, R. B.; CARVAlHAES, F. F. D. Psicologia e políticas públicas: impasses e reinvenções. Psicologia \& Sociedade, v. 28, n. 2, p. 247-256, 2016.

SILVA, R. B.; BONATTI, G. L. Reflexões sobre a clínica ampliada nos Centros de Referência da Assistência Social (CRAS). Psicologia Revista, v. 28, p. 379-394, 2019.

SOUZA, J.; KANTORSKI, P. L.; LUIZ, M. A. V. Análise documental e observação participante na pesquisa em saúde mental. Revista Baiana de Enfermagem, Salvador, v. 25, n. 2, p. 221-228, 2011.

SOUZA, T. Redução de Danos no Brasil: a clínica e a política em movimento. 116f. Mestrado em Psicologia. Universidade Federal Fluminense, Niterói, 2007. Disponível em: https://app.uff.br/slab/uploads/2007_d_Tadeu.pdf.Acessado. Acesso em: 5 jul. 2020

SUNDFELD, A. C. Clínica ampliada na atenção básica e processos de subjetivação: relato de uma experiência. Physis: Revista de Saúde Coletiva, v. 20, n. 4, p. 1079-1097, 2010.

\footnotetext{
' Doutor em Educação pela Universidade Estadual Paulista Júlio de Mesquita Filho e Docente do Departamento de Psicologia Social e Institucional e Programa de Pós-Graduação em Psicologia da Universidade Estadual de Londrina - UEL. Email: tibx211@yahoo.com.br

ii Graduanda do curso de Psicologia da Universidade Estadual de Londrina - UEL. Email: mianiluiza@gmail.com

iii Graduanda do curso de Psicologia da Universidade Estadual de Londrina - UEL. Email: grabonatti1580@gmail.com
} 